\title{
Interactive comment on "Evaluation of aerosol and cloud properties in three climate models using MODIS observations and its corresponding COSP simulator, and their application in aerosol-cloud interaction" by Giulia Saponaro et al.
}

Anonymous Referee \#2

Received and published: 14 November 2019

In this work three different atmospheric models (ECHAM-HAM, ECHAM-HAM-SALSA and NorESM) are compared against MODIS retrievals by using the COSP instrument simulator. Emphasis is given on the estimation of cloud droplet number concentration (CDNC) and its sensitivity to aerosol load, as a way to assess the importance of aerosol-cloud interactions. The paper is well written and the subject is of relevance to the atmospheric community. In general the paper is correct and I don't see any major flaw that would limit publication. At the same time it is hard to tell what original contribution this paper offers, other than a throughout description of the models performance. 
In that sense I think this work would be more suitable for a journal like Geophysical Model Development. If the authors still want to publish it in ACP a deeper exploration of the observed differences in CDNC-aerosol index sensitivity must be included. I have made some suggestions below.

\section{Comments}

The authors simply mention that the MODIS-derived CDNC and the direct model output are different, which is true, but offer no explanation of why those difference appear. Intuitively, the authors assume that the CDNC at cloud top should correspond to the MODIS retrieval, but omit the fact that MODIS does not produce CDNC directly. Instead using the method from Bennartz et al . (2007) one may argue that CDNC at cloud base or even the maximum CDNC in the column should be used. Also since the assumption of adiabaticity and vertically constant CDNC is embedded in the MODIS-derived CDNC the authors should limit the analysis only to regions where those assumption are valid (probably mostly over ocean).

The global CNDC-aerosol index sensitivity calculations (what the authors mistakenly call $\mathrm{ACl}$ throughout the manuscript) are also mostly descriptive and even though some speculation is given on the possible causes for discrepancy I imagine there is enough model output to go deeper (see specific comments). Also, in principle calculating the "ACl" from 2D vertically integrated fields makes little sense. It is not clear what role the assumptions of overlap are, or even whether the cloud and the aerosol occupy the same space.

Finally there is the issue of "the ACl". Aerosol-cloud interactions encompass many processes occurring in clouds as a result of the presence of aerosols. As a noun, aerosolcloud interactions is an area of study, not a metric. So it is troubling, and in many places grammatically incorrect, that $\mathrm{ACl}$ are reduced to a single number and equated to the CDNC-AI sensitivity. Please correct this and be precise in the terminology used. 
Page 3, Line 34. Why 2008? The horizontal resolution is low enough that it should be easy to run a couple of years at least. MODIS data spans at least 15 years as well.

Page 3, Line 35. No, the aerosol- cloud interaction is not a metric, and certainly not confused with the aerosol indirect effect. Even saying "the aerosol cloud interaction" is probably incorrect. Moreover, $\mathrm{ACl}$ is referred here as a metric and later on as a topic. Please be precise in the terms used.

Page 4 Line 32. This is the CDNC-Al sensitivity. Also I am not convinced that the meteorological component is completely removed, please explain.

Page 4 Line 32. Why should the liquid water path remain constant? Is there a clear connection between the CDNC-AI sensitivity and the first aerosol indirect effect?

Page 9, Line 17. Why is the vertical resolution different than when running ECHAMHAM?

Page 11, Line 6-8. This seems incorrect and could be a major flaw. COSP should account for the fact that MODIS only sees in-cloud values. It makes little sense to divide two column-integrated, $2 \mathrm{D}$ values. If COSP cannot account for it, then the $3 \mathrm{D}$ model calculation should be converted to in-cloud values before sending it to COSP to calculate MODIS-like values. Please clarify.

Page 11, Line 18. Is there a MODIS algorithm to retrieve CDNC? It may be more correct to say in MODIS cloud effective radius is biased towards cloud top values, which may propagate to the CDNC calculation. The method used to estimate CDNC (equation 1) should approximate better cloud-base values.

Page 12, Section 3.2. Please label this equation, and explain the assumptions behind it. Given than clouds are 3D, to what vertical level should this calculation correspond? Also, if this applies well in the stratiform marine boundary layer why are the authors applying it globally? What would be the error incurred in applying it to a shallow convective trade cumulus for example? 
Page 12, Line 15. This is the CDNC-Al sensitivity, not "the ACl".

Page 12, Lines 16-28. Please expand this. Is this done using a linear regression of the ACPD CDNC and Al daily time series? Given that this is a global calculation why would the number of data points be different? Also, it is not clear what the "ACl" from 2D vertically integrated fields represents (see general comments).

Page 13, Line 18. Does COSP produce COP CF or CF all? Maybe the discussion should be limited to that one.

Page 15, Lines 1-10. This is very vague and should be explored in more detail (see comments above).

Page 15, Lines 17-20. Is the difference due to the activation scheme or to the aerosol models?

Page 15, Lines 25-30. These two sentences contradict each other.

Page 15, Lines 30-35. This is purely speculative.

Page 17, Line 15-25. Could this be corroborated? It seems odd that the sensitivity would be negative.

Page 17, Line 25. Global maps of CDNC-AI sensitivity should show this better.

Interactive comment on Atmos. Chem. Phys. Discuss., https://doi.org/10.5194/acp-2019-631, 2019. 